\title{
3D Trench Electrode Si Detector with Adjustable Central Collection Electrode
}

\author{
Chuan Liao ${ }^{1,2,3, a}$, Ya Zhang ${ }^{1,2,3, b}$, Xiaojie Liu ${ }^{1,2,3, \mathrm{c}}$ and Zheng $\mathrm{Li}^{1,2,3^{*}}$ \\ ${ }^{1}$ School of Materials Science and Engineering, Xiangtan University, Xiangtan 411105, China \\ ${ }^{2}$ Center for Semiconductor Particle and photon Imaging Detector Development and Fabrication, \\ Xiangtan University, Xiangtan 411105, China \\ ${ }^{3}$ National-Provincial Laboratory of Special Function Thin Film Materials, and School of Materials \\ Science and Engineering, Xiangtan University, Hunan 411105, China \\ a270883847@qq.com, ${ }^{b} 447604418 @ q q . c o m,{ }^{c^{*}}$ zhengli58@gmail.com
}

\begin{abstract}
Keywords: 3D-TCAD, Adjustable, Depletion regions, Electrical characteristics.
Abstract. In order to improve the radiation resistance of semiconductor detector, 3D trench electrode Si detector structures have been proposed by BNL at the end of 2009. In this paper, we propose a 3D trench electrode Si detector with adjustable central collection electrode, which integrates advantages of cylindrical units and parallel units. For those combine units, we use 1D Poisson equation as the first order approximation to study the changing of depletion in different regions. These results have been compared to those obtained from the full 3D simulation using a 3D-TCAD tool. According to our calculations, we designed a 3D trench electrode Si detector with adjustable central collection electrode. Furthermore electric characteristics of this type of detectors with different lengths of central collection electrode $\left(l_{p+}\right)$ have been simulated using the 3D-TCAD.
\end{abstract}

\section{Introduction}

Silicon detector, widely used in high energy and nuclear physics experiments, suffer severe radiation damages that lead to degradations in detector performance. These degradations include significant increases in leakage current, bulk resistivity, space charge concentration, and free carrier trapping. For LHC upgrade, HL-LHC, the radiation environment will become more severe, and even more radiation tolerant detectors will be required.

In order to improve the detector radiation tolerance, in the 1997, the conventional 3D column electrode detector was propose by Parker at University of Hawaii [1,2]. It is named from the 3D-process, $\mathrm{p}^{+}$and $\mathrm{n}^{+}$electrode columns with typical diameter of $10 \mu \mathrm{m}$ are etched into the detector thickness. The electrode spacing ( $\lambda$, the distance between neighboring $\mathrm{p}^{+}$and $\mathrm{n}^{+}$electrode columns. As shown Fig.1(a), $\lambda_{x}$ and $\lambda_{y}$ ) can be made in the order of $30-50 \mu \mathrm{m}$, which provide small full depletion voltage and much reduced carriers trapping [3.4].

Since the invention of 3D column electrode detectors, continuous advances of the detector structure have been made. In 3D column electrode detectors, there exist regions of non-homogeneous electric field [5], which may get worse in heavy irradiation environment. In 2009, the 3D trench electrode Si detector structure had been proposed [6]. Because 3D trench electrode Si detector structures have more uniform electric field, it is superior as compared to 3D column electrode detector in radiation tolerance. Although it has many advantages in performance for high-energy physics applications, the cell size of a 3D trench electrode Si detector with a column central electrode is limited by the electrode spacing. As radiation fluence increases (now up to $10^{16} \mathrm{n}_{\mathrm{eq}} / \mathrm{cm}^{2}$ ), one has to decrease the cell electrode spacing $\lambda$ to meet the radiation hardness requirements. This in turn will diminish the detector cell size of these types of 3D trench electrode Si detectors, since the cell size is in the order of $\pi \lambda^{2}$ (As electrode spacing increase, full depletion voltage obviously increase and charge collection efficiency (CCE) is significantly decline) [7].

In the 2009, Li et al mentioned a square shape of 3D trench electrode Si detector with parallel electrode(parallel plates) [6], no detail of design and simulations were given. Also, square shape 
detector has more electric field non uniformity. In this paper, based on their study, we studied the detector full depletion voltages $\left(V_{f d}\right)$ in different directions ( $\mathrm{x}$ and y directions in Fig.1(a)), and proposed a better cell structure (combining a cylindrical unit and a parallel plate unit as the trench electrode, and an "adjustable central collection electrode"). Furthermore electrical characteristics of this type of detectors with different lengths of central collection electrode have been simulated using the 3D-TCAD. The detail information will be presented on following paper.

\section{Design theory}

A cell of 3D trench electrode Si detector with adjustable electrode has been presented in this paper, as Fig.1(a) show. In Fig.1(a), the detector height D is $302 \mu \mathrm{m}$, the donor doping concentration of $\mathrm{n}^{+}$ trench $N_{D}$ is $10^{19} \mathrm{~cm}^{3}$, and the acceptor doping concentration of $\mathrm{p}^{+}$central $N_{A}$ is $10^{19} \mathrm{~cm}^{3}$. In order to improve the detector's radiation resistance [8], the bulk regions (between $\mathrm{p}^{+}$and $\mathrm{n}^{+}$) and $\mathrm{P}$-type $\mathrm{Si}$ bottom is doped in a concentration of $10^{12} \mathrm{~cm}^{3}$, which is called the effective doping concentration $N_{\text {eff. }}$. Due to the processing limitation, the height of electrode is set as $270 \mu \mathrm{m}$, the length of silicon substrate is $30 \mu \mathrm{m}$ [6], and the thickness of $\mathrm{p}^{+}$or $\mathrm{n}^{+} \tau$ is $10 \mu \mathrm{m}$. Under the P-type silicon substrate, there is a $\mathrm{SiO}_{2}$ whose thickness is $1 \mu \mathrm{m}$. Aluminum layer with a thickness of $1 \mu \mathrm{m}$ is put on the surface of $\mathrm{p}^{+}$and $\mathrm{n}^{+}$. $\mathrm{SiO}_{2}$ layer with a thickness of $1 \mu \mathrm{m}$ is put on the surface of bulk.

As shown in Fig.1(a), the electrode spacing in x-direction $\left(\lambda_{x}\right)$ is between two parallel plate electrodes and that in y-direction $\left(\lambda_{y}\right)$ is between the semi-cylindrical trench electrodes in the two cell ends and ends of the central electrode. The relationship between $\lambda_{x}$ and $\lambda_{y}$ is important for the new structure to obtain a near homogeneous full depletion voltage in all detector regions. The full depletion voltages, and their dependencies on $\lambda_{x}$ and $\lambda_{y}$, in two different regions can be obtained from solving Poisson equations with different boundary conditions (e.g. linear and cylindrical boundaries, respectively). The approach here is that by choosing the same full depletion voltages for two different regions to design an optimum detector cell. As limited of paper length, only result has be given:

(1)The dependence of the full depletion voltage in $\mathrm{x}$ direction $V_{f d, x}$ on the electrode spacing $\lambda_{x}$ (linear boundaries):

$$
V_{f d, x} \cong \frac{e N_{e f f} \lambda_{x}{ }^{2}}{2 \varepsilon \varepsilon_{0}}-V_{b i}
$$

Where $N_{\text {eff }}$ the effective doping concentration in the p-type cell bulk and bottom, $V_{b i}$ is the build-in potential.

(2)The dependence of the full depletion voltage in y direction $V_{f d, y}$ on the electrode spacing $\lambda_{y}$ (cylindrical boundaries):

$$
V_{f d, y} \cong \frac{e N_{e f f}}{4 \varepsilon \varepsilon_{0}}\left[\left(\lambda_{y}+\frac{\tau}{2}\right)^{2}-\left(\frac{\tau}{2}\right)^{2}\right]-\frac{e N_{e f f}}{2 \varepsilon \varepsilon_{0}}\left(\frac{\tau}{2}\right)^{2} \ln \left(\frac{\lambda_{y}+\frac{\tau}{2}}{\frac{\tau}{2}}\right)-V_{b i}
$$

(3)The dependence of $\lambda_{x}$ on $\lambda_{y}$ can be obtained:

$$
\lambda_{x}=\sqrt{\frac{1}{2}\left[\left(\lambda_{y}+\frac{\tau}{2}\right)^{2}-\left(\frac{\tau}{2}\right)^{2}\right]-\left(\frac{\tau}{2}\right)^{2} \ln \left(\frac{\lambda_{y}+\frac{\tau}{2}}{\frac{\tau}{2}}\right)}
$$

In this paper, we set value of $\lambda_{y}$ as $60 \mu \mathrm{m}$. According to Eq.3, a result of $\lambda_{x}=45 \mu \mathrm{m}$ can be obtained. TCAD tool has been used to simulate hole concentration for the changing of depletion depth. Since $10^{12} \mathrm{~cm}^{-3}$ (In the figure, it is shown as 12 since it is a $\log$ scale) was defined as a upper limit of hole 
concentration in simulated result (Fig. 1b), the reddest regions in the bulk is not depleted. As the Fig.1(b) shown, full depletion voltage in about $2.5 \mathrm{~V}$, Calculated result is $2.2 \mathrm{~V}$, which is similar with simulations. Simulations results also show full depletion voltage is similar between parallel $\mathrm{x}$ direction and y direction. At last, the approach of model design has been give by Eq.3, which meets our requirement. The following section, full $3 \mathrm{D}$-simulation is used to simulate electrical characteristics (including electric potential and electric field) of those cells.

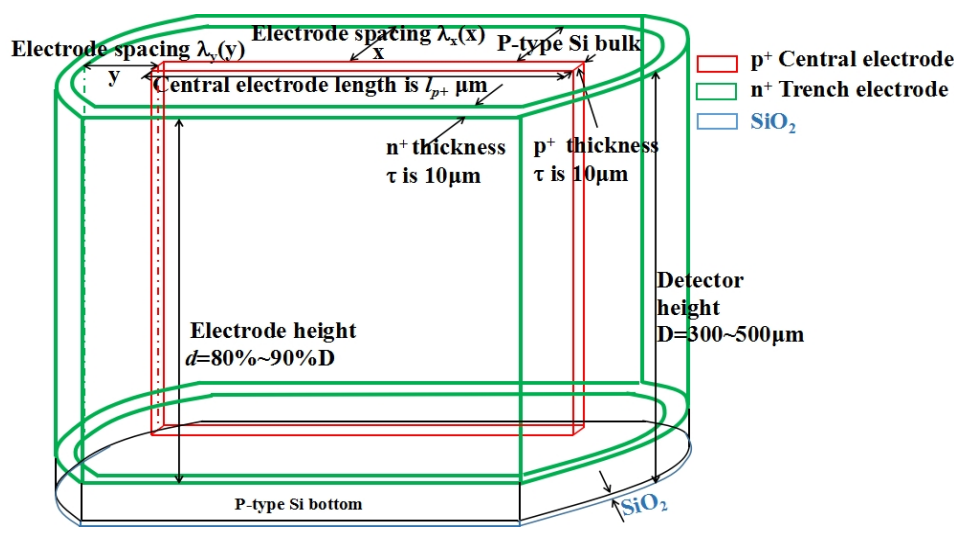

(a)

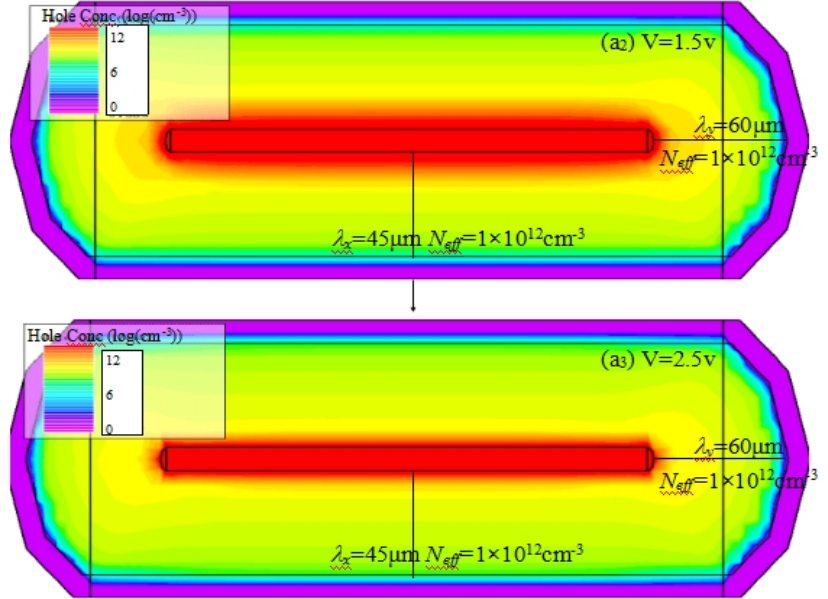

(b)

Fig.1 (a)3D trench Si detector with adjustable central collection electrode. (b)Hole concentration at $\mathrm{D}_{Z}=165 \mu \mathrm{m}$

\section{Electric potential}

Full 3D simulations have also been performed with full boundary conditions on both surfaces with oxide charges $\left(4 \times 10^{11} \mathrm{~cm}^{-3}\right)$. Due to paper length limitation, only a few of results have been shown. In this paper, TCAD tools are used to simulate electric potential of detector units with different $l_{p+}$. The electrode spacing: $\lambda_{x}=45 \mu \mathrm{m}$ and $\lambda_{y}=60 \mu \mathrm{m}$.

The advantage of the structure is adjustable:

$$
L=2 \lambda_{y}+l_{p^{+}}+2 \tau
$$

where $L$ is the length of unit. As shown in Fig.2, the distribution of potential is like a funnel, the

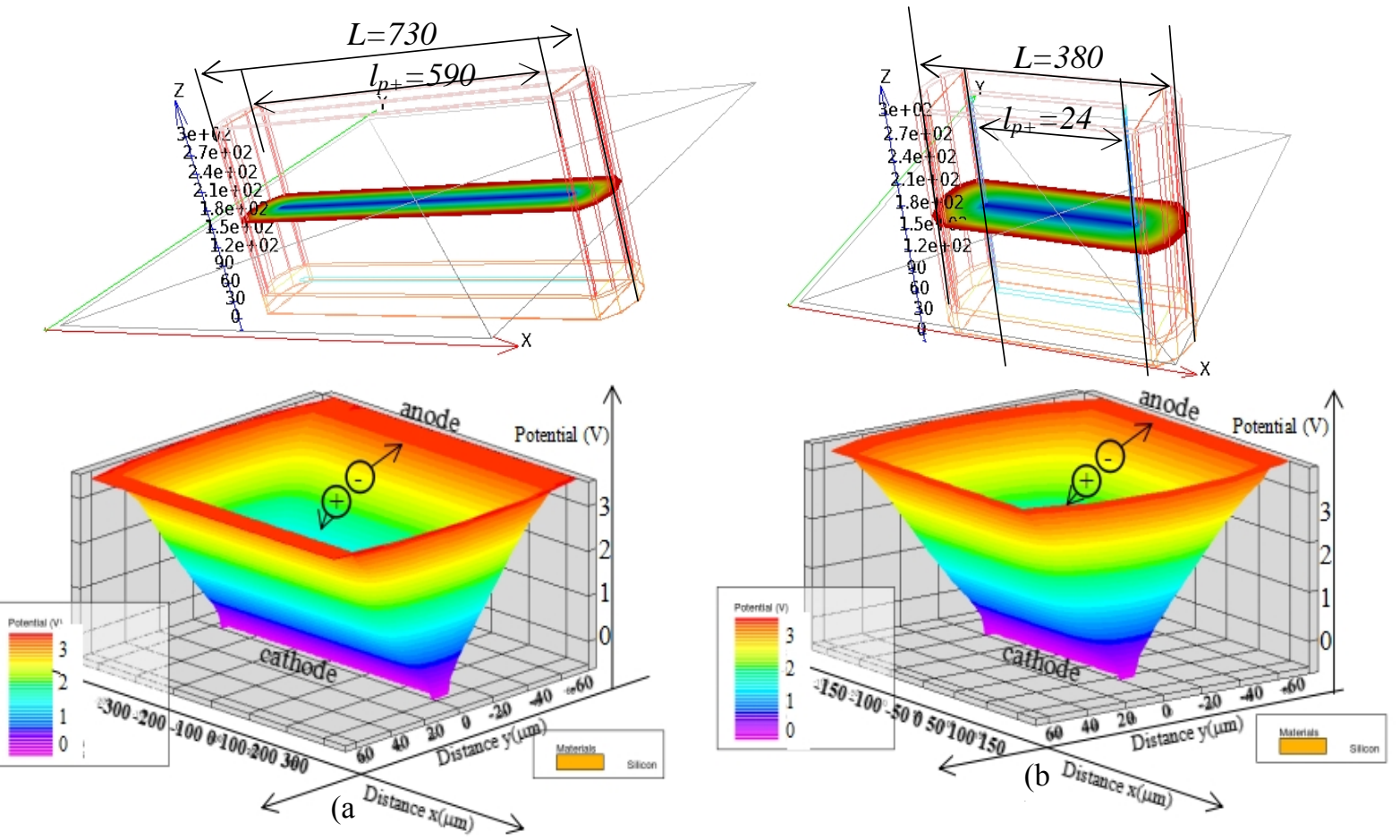

Fig. 2 cross section of potential with different central collection electrode, $D_{z}=165 \mu \mathrm{m}$.

(a) $l_{p+}=590 \mu \mathrm{m}$; (b) $l_{p+}=240 \mu \mathrm{m}$ 
electron and hole are moving along the gradient to the anode and cathode, respectively. A distribution of potential isn't impacted by $l_{p+}$ extending $\left(l_{p+}>>\lambda_{x, y}\right)$.

\section{Electric field}

In order to prove the advantage of our approach, electric field of the designed structure (Fig.2a) is compared with other structure that $l_{p+}$ has been shortened ( $\lambda_{\mathrm{y}}$ has been lengthen). As Fig.3 shows, the purple region is low electric field. Fig.3(a) is the designed structure by our approach and electric potential has been shown on Fig.2(a) $\left(l_{p+}=590 \mu \mathrm{m}, \mathrm{L}=730 \mu \mathrm{m}\right)$. Fig.3(b) is the compared structure's electric field distribution with $l_{p+}=570 \mu \mathrm{m}$ (also $\mathrm{L}=730 \mu \mathrm{m}$ ). As Fig.3(b) shows, low electric field area increases significantly as $\lambda_{\mathrm{y}}$ is just increased $10 \mu \mathrm{m}$ from the value shown in Fig.3(a). According to comparison, a conclusion can be obtained: for full depletion in all regions, a theory value of $\lambda_{y}$ (given by Eq.3) is longest length without low electric field in bulk.

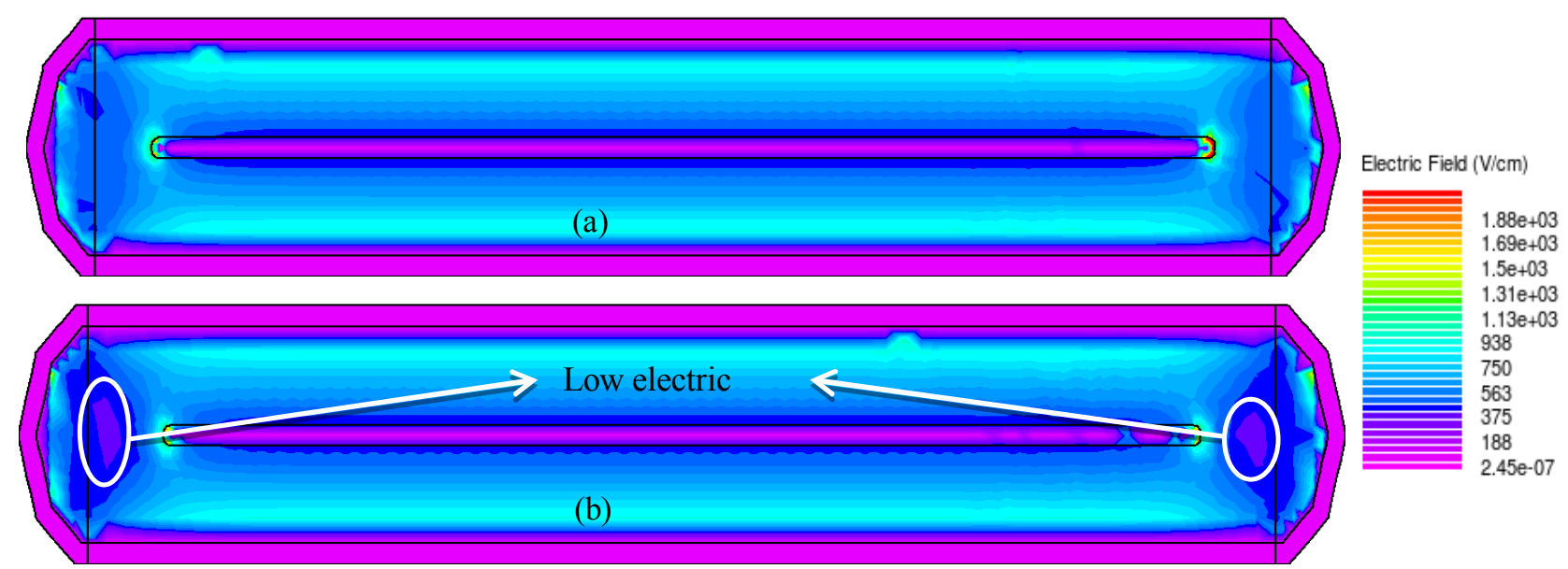

Fig. 3 Cross section of electric field, at $\mathrm{D}_{\mathrm{z}}=165 \mu \mathrm{m}$, under full depletion voltage.

(a) $l_{p+}=590 \mu \mathrm{m}, \lambda_{y}=60 \mu \mathrm{m}$ (b) $l_{p+}=570 \mu \mathrm{m}, \lambda_{y}=70 \mu \mathrm{m}$

\section{Conclusions}

As demonstrated in the paper, we derived Eq.3 to define a $\lambda_{x}$ to insure the same full depletion voltages of two different regions in our detector structure. Thus the relationship of $\lambda_{x}$ and $\lambda_{y}$ can be obtained. According to calculations, 3D trench electrode Si detector with adjustable central collection electrode has been designed and full depletion voltage $V_{f d}=2.2 \mathrm{~V}$ has been obtained at $N_{\text {eff }}=10^{12} \mathrm{~cm}^{-3}$, and this result is similar with 3D full-simulations. As the full 3D simulations shows, our new detector structure can effectively enlarge the detector unit cell without introducing low electric field region in the cell.

\section{References}

[1] S. Parker, C. Kenney, J. Segal, Nucl. Instr. and Meth. A 395 (1997) 328.

[2] C.J. Kenney, S.I. Parker, J. Segal, C. Storment, IEEE Trans. Nucl. Sci. NS46 (4) (1999) 1224.

[3] Zheng Li, M. Abreu, P. Anbinderis, et al., Nucl. Instr. and Meth. A572 (2007) 305.

[4] C. Piemonte, M. Boscardin, G.F. Dalla Betta, S. Ronchin, N. Zorzi, Nucl. Instr. and Meth. A 541 (2005) 441.

[5] Tanja Grönlund, Zheng Li, Gabriella Carini, Michael Li, Nucl. Instr. and Meth. A 586 (2008) 180.

[6] Zheng Li. Nucl. Instr. and Meth. A 658(2011) 90.

[7] Hao Ding, Jianwei Chen, Zheng Li, Shaoan Yan. Nucl. Instr. and Meth. A 796 (2015) 29.

[8] S. Pirollo et al., Nucl. Inst. \& Meth., A426, 126-130 (1999) 